\title{
Genetic Analysis of Superior Crosses for Quantitative Traits in Sesame (Sesamum indicum L.)
}

\author{
J. Jawahar Lal ${ }^{1 *}$, Kuldeep Singh Dangi ${ }^{2}$ and S. Sudheer Kumar ${ }^{2}$
}

${ }^{1}$ Indian Institute of Oilseeds Research, Rajendranagar, Hyderabad-30, India

${ }^{2}$ Department of Genetics and Plant Breeding, College of Agriculture, PJTSAU, Rajendranagar, Hyderabad - 500 030, Andhra Pradesh, India

*Corresponding author

\section{A B S T R A C T}

\section{Keywords}

Sesame, per se, Heterosis, gca effect, sca effect, Genetic analysis, Superior crosses

Article Info

Accepted:

20 December 2019

Available Online:

20 January 2020
Sixteen genotypes consisting of ten lines and six testers were crossed in line $\mathrm{x}$ tester fashion. The hybrids and their parents were used to estimate three types of heterosis, sca effects for 11 quantitative traits. Based on the sca, heterosis and per se performance, the superior crosses were identified for each trait. The crossesPKDS-62 x IS $562 \mathrm{~B}$ and KKS-98049 x KMR-78 which showed superior performance in yield and yield contributing traits and these were the best among the 60 crosses studied. These crosses may be used for commercial exploitation of heterosis in sesame.

\section{Introduction}

Sesame (Sesamum indicum L.) is one of the oldest oil seed crops known and used by man. Regarded as the "Queen of oil seeds", oil extracted from sesame is among the highly prized few edible oils by virtue of its quality. The antioxidants sesamin and sesmolin increase the oil quality by making it resistant to rancidity. Sesame seed is rich in oil (50$53 \%$ ) and protein (20-26\%) on an average. Sesame oil is semidrying and most stable against oxidative rancidity and used in various industries besides in cooking. The oil is also rich in vitamin-E and contains an essential amino acid methionine (3.4\%) (Mosjidis 1982).

Myanmar, India and China are the top three sesame producing countries in the world. But, the productivity of sesame in India is very low (432 kg/ha) compared to China (1382 $\mathrm{kg} / \mathrm{ha}$ ) and Myanmar (565 kg/ha) (FAO, 2017). Though sesame is predominantly selfpollinated crop, considerable cross pollination has been recorded. The epipetalous nature of stamens and high heterosis will pave the way to development of hybrid sesame. In this 
study an attempt has been made to find the level of heterosis and identification of superior crosses for heterosis exploitation.

Hybrid sesame is an optimistic approach for significant improvement in genetic potential for yield and yield attributing traits. For commercial exploitation of heterosis in sesame, the pre-requisites are identification of parents which show good heterosis on crossing and production of hybrids with low cost. The work on heterosis has been reviewed from time to time on yield and yield components in sesame. Thus, the aim of heterosis analysis is to find out the best combination of parents for their prospects for future use in hybrid breeding programme. In the present investigation, heterosis was measured over mid parent, better parent and standard check (Swetha til) for yield and yield components. A high level of heterosis and sca effects in desired direction was observed in several hybrids for various characters.

\section{Materials and Methods}

Ten elite high yielding sesame genotypes collected from different agro morphological regions, India as female parents ( IS $1547 \mathrm{~A}$, KKS-98049, PKDS-62, SI-7818, JCS-720, JCS-724, KMR-108, KMR-24, S-0018 and CST 2001-5) and six genotypes as male parent (KMS 5-396, JCS-507, IS 562 B, SI3171, KMR-78 and TKG-22) in a line $\mathrm{x}$ tester mating design. These selected parents were crossed in $\mathrm{L} \times \mathrm{T}$ fashion to generate 60 hybrids in Kharif, 2007. These 60 crosses along with their parents and checks were evaluated randomized block design with three replications at college farm, college of agriculture, ANGRAU, Rajendranagar, Hyderabad during Rabi, 2007-08, 2008-09. Each genotype in each replication was sown by dibbling the seeds in four rows of plot $4 \mathrm{~m}$ length, with a spacing of $30 \mathrm{~cm}$ between the rows and $10 \mathrm{~cm}$ between the plants. The recommended packages of practices were adopted to raise a healthy crop. Plant protection measures were taken up as and when required. The genotypes were harvested as and when attained physiological maturity.

Morphology of all sesame genotypes were characterized by 11 quantitative traits on ten randomly tagged plants in each genotype. The traits measured were number of days to $50 \%$ flowering, numbers of days to maturity, number of effective primaries per plant, plant height $(\mathrm{cm})$, total number of capsules per plant, capsule length $(\mathrm{cm})$, number of seeds per capsule, 1000-seed weight (g), oil content (\%), chlorophyll content (SPAD units) and seed yield per plant $(\mathrm{g})$. The mean of ten plants for all the characters, except days to $50 \%$ flowering and days to maturity was utilized for carrying out statistical analysis. For days to $50 \%$ flowering and days to maturity was recorded on plot basis. Line $\mathrm{x}$ tester analysis was carried out according to Kempthorne 1957. The heterosis was estimated over the check as per the standard procedure. Mean values per replication for all traits were subjected to analysis of variance according to Panse and Sukhatme (1985) for randomized block design. The estimates of general and specific combining ability and their variances were obtained by using covariance of half sibs and full sibs.

\section{Results and Discussion}

A comparison of five best cross combinations for various characters is presented in Table 1. The perusal of sca effects of different characters in present investigation revealed that sca effects and per se performance of the crosses were closely related, which indicates that the hybrids with high per se performance need be the one with high sca effects and vice versa. But for the character, days to 50 per cent flowering (desirable in negative direction) high correlations between the sca 
effects and per se performance of the $\mathrm{F}_{1}$ crosses was observed, which signifies the contribution of additive gene effects in the inheritance of these traits. So the cross combinations may be selected either on the basis of sca or mean performance or in combination.

With regard to 1000 seed weight significant positive $s c a$ effects observed in twelve (12) hybrids. The crosses JCS-720 x KMS 5-396 (low x high), CST 2001-5 x IS 562B (high x low), KKS-98049 x JCS-507(low $x$ low) involved parents of high $\mathrm{x}$ low, low $\mathrm{x}$ high and low $\mathrm{x}$ low indicating the presence of genetic diversity among the parents. The trait oil content recorded significant $s c a$ effects in 24 crosses in positive direction. The crosses SI-7818 x SI-3171(high x low), KMR-108 x KMR-78 (low $x$ high), PKDS-62 x JCS507(low $x$ low) were the good specific combiners for oil content. Presence of at least one parent with high gca effects indicating that a high general combiner in the cross combination might result in good specific combination.

For the trait chlorophyll content eight crosses recorded significant positive effects.The crosses JCS-724 x SI-3171 (low x high), S0018 x KMR-78 (high x low), KMR-108 x TKG-22 (low $\mathrm{x}$ low) exhibited significant positive sca effects. The trait oil content recorded significant sca effects in 24 crosses in positive direction. The crosses SI-7818 $\mathrm{x}$ SI-3171(high x low), KMR-108 x KMR-78 (low x high), PKDS-62 x JCS-507(low x low) were the good specific combiners for oil content. Presence of at least one parent with high $\mathrm{gca}$ effects indicating that a high general combiner in the cross combination might result in good specific combination.

The highest sca effects for seed yield are observed in 11 crosses viz., PKDS-62 x IS562 B (low x low), KKS-98049 x KMR-78 (low x low), CST 2001-5 x TKG-22 (high x low), SI-7818 x SI-3171 (high x low), KKS98049 x SI-3171 (high x high). Almost all types of combinations produced superior hybrids for seed yield per plant. The first five crosses which recorded high sca effects for yield also exhibited significant sca effects in desired direction for important yield contributing traits viz., days to 50 per cent flowering, plant height, number of effective primaries per plant, number of capsules per plant, capsule length, seeds per capsule, 1000 seed weight and oil content indicating the possibility of exploiting these cross combinations for yield improvement in sesame through heterosis breeding. Interestingly, except IS 1547 A x KMS 5-396 these five cross combinations exhibited non significant sca effects for oil content. This suggested that high yielding hybrids with acceptable levels of quality traits should be considered. Of the eleven hybrids exhibited positive $s c a$ effects for yield, none exhibited significant sca effects for oil content except the cross combination PKDS-62 x IS 562 B, IS-1547 A x KMS 5-396, CST 2001-5 x TKG-22 and KKS-98049 x JCS-507 which recorded desirable $s c a$ effects for oil content.

High sca effects observed in the crosses for the characters studied were either due to high $\mathrm{x}$ high, high $\mathrm{x}$ low, low $\mathrm{x}$ high and low $\mathrm{x}$ low combining parents. The ideal specific combination should be the one where high magnitude of sca in addition to high $\mathrm{gca}$ in both or at least one of the parents is present. Similar results of high $\mathrm{x}$ low / low x high, low $\mathrm{x}$ low has been reported by earlier workers Neelima et al., (2004) and Rauf et al., (2006).

Significant but negative heterosis over mid parent and better parent was recorded in eleven and twenty five crosses respectively for days to 50 per cent flowering. While three crosses were found to be significantly earlier than the standard check. Negative heterosis is 
considered to be desirable since earliness is preferred over later flowering in different situations. Negative heterosis over mid parent was reported by Saravanan and Nadarajan (2002), Karad et al., (2002) and Raghunaiah (2005) reported significant negative heterosis over commercial check.

Sixteen crosses exhibited significant negative heterosis over mid parent for days to maturity whereas only twenty seven crosses recorded significant negative heterosis for better parent and seven crosses recorded negative heterosis for standard check. Similar results over mid parent are being reported by Mishra and Sikarwar (2001), Nijagun et al.,(2003)and over better parent by Raghunaiah (2005).

More number of crosses (25) exhibited significant positive heterosis over mid parent for number of effective capsules per plant compared to heterosis over better parent (12 crosses). The crosses IS 1547 A x KMS 5396, CST 2001-5 x KMS 5-396 and KMR$108 \times$ JCS-507 recorded the highest positive significant heterosis over both mid and better parents, whereas five crosses recorded significant positive heterosis over the check. The crosses SI-7818 x KMS 5-396 and SI$7818 \times$ SI-3171 found to exhibit all three types of heterosis considerably. Significant positive heterosis over standard check is in agreement with the findings of Mothilal and Manoharan (2004)

Significant positive heterosis over mid parent, better parent and standard check was observed in eleven, five and thirteen crosses respectively for number of seeds per capsule. The crosses KMR-108 x IS 562 B observed to be exhibit both heterobeltiosis and average heterosis. The cross JCS-724 x SI-3171, CST 2001-5 x JCS-507, CST 2001-5 x IS 562 B and KMR-24 x KMS 5-396 found to exhibit all three types of heterosis considerably. Relative heterosis has also been reported by
Mothilal and Ganesan (2005).

With regards to 1000 seed weight significant positive sca effects observed in twelve hybrids and seven crosses recorded significant and positive mid parental heterosis, whereas five and twelve hybrids recorded heterobeltiosis and standard heterosis respectively. The crosses JCS-720 x KMS 5396, CST 2001-5 x IS 562 B and KKS-98049 $\mathrm{x}$ JCS-507 recorded considerable heterosis over mid parent, better parent and standard check. Significant positive heterosis over better parent is being reported by Ray and Sen (1992).

In case of oil content twenty one and seven crosses exhibited significant and positive heterosis over mid parent and better parent respectively, whereas none of the crosses recorded significant positive heterosis over standard check. The hybrids S-0018 x TKG22, JCS-720 x IS 562 B, S-0018 x IS 562 B, JCS-720 x TKG-22, KMR-24 x TKG-22, KMR-24 x IS 562 B and SI-7818 x SI-3171 exhibited heterosis over mid parent and better parent. Positive heterosis over mid parent was reported by Deepa and Ananda (2001) and significant positive heterosis over better parent and commercial check is given by Raghunaiah (2005).

For seed yield per plant significant positive heterosis was observed in 27 crosses over mid parent, 16 crosses over better parent and 5 crosses over standard check. The crosses SI7818 x SI-3171, PKDS-62 x IS 562 B, KKS98049 x SI-3171 and KKS-98049 x KMR-78 were exhibited considerable high heterosis over mid parent, better parent and standard check, while the crosses IS 1547 A x KMS 5396 and JCS-724 x SI-3171 were recorded highest heterosis over both mid parent and better parent. 
Table.1 Analysis of variance for combining ability for yield and yield component characters in sesame

\begin{tabular}{|c|c|c|c|c|c|c|c|c|c|c|c|c|}
\hline $\begin{array}{l}\text { Source of } \\
\text { variation }\end{array}$ & $\begin{array}{l}\text { Degrees of } \\
\text { freedom }\end{array}$ & $\begin{array}{c}\text { Days to } \\
50 \% \\
\text { flowering }\end{array}$ & $\begin{array}{l}\text { Days to } \\
\text { maturity }\end{array}$ & $\begin{array}{c}\text { Plant } \\
\text { height } \\
\text { (cm) }\end{array}$ & $\begin{array}{c}\begin{array}{c}\text { No. of } \\
\text { effective } \\
\text { primaries/ } \\
\text { plant }\end{array} \\
\end{array}$ & $\begin{array}{l}\text { No. of } \\
\text { effective } \\
\text { capsules/ } \\
\text { plant }\end{array}$ & $\begin{array}{c}\text { Capsule } \\
\text { length } \\
(\mathrm{cm})\end{array}$ & $\begin{array}{c}\text { No. of } \\
\text { seeds/ } \\
\text { capsule }\end{array}$ & $\begin{array}{l}1000- \\
\text { seed } \\
\text { weight } \\
(\mathrm{g})\end{array}$ & $\begin{array}{c}\text { Seed } \\
\text { yield/ } \\
\text { plant (g) }\end{array}$ & $\begin{array}{c}\text { Oil } \\
\text { content } \\
(\%)\end{array}$ & $\begin{array}{c}\text { Chlorophyll } \\
\text { content } \\
\text { (SPAD } \\
\text { Units) }\end{array}$ \\
\hline Replications & 2 & 0.96 & 1.45 & 0.96 & $0.19 *$ & 78.733 & 0.03 & 17.046 & 0.056 & 0.24 & 0.098 & 9.73 \\
\hline Treatments & 75 & $10.96 * *$ & $20.58 * *$ & $263.23 * *$ & $6.59 * *$ & $1564.857 * *$ & $0.06 * *$ & $125.683^{* *}$ & $0.281 * *$ & $16.93 * *$ & $29.057 * *$ & $56.71 * *$ \\
\hline Parents & 15 & $24.74 * *$ & $22.17 * *$ & $158.63 * *$ & $2.45^{* *}$ & $1079.83 * *$ & $0.05^{* *}$ & $100.09 * *$ & $0.34 * *$ & $13.73 * *$ & $50.70 * *$ & $69.24 * *$ \\
\hline $\begin{array}{l}\text { Parents vs } \\
\text { Crosses }\end{array}$ & 1 & 1.07 & $5.13 * *$ & $189.16^{* *}$ & $22.98 * *$ & $4224.06 * *$ & $0.11^{*}$ & $58.82 * *$ & $0.43 * *$ & $85.80 * *$ & $56.54 * *$ & $416.82 * *$ \\
\hline Crosses & 59 & $7.61 * *$ & $20.44 * *$ & $291.08 * *$ & $7.37 * *$ & $1643.10^{* *}$ & $0.06 * *$ & $133.30 * *$ & $0.26 * *$ & $16.57 * *$ & $23.08 * *$ & $47.42 * *$ \\
\hline Lines & 9 & $18.29 * *$ & 20.71 & $703.66^{* *}$ & 8.67 & 2533.13 & $0.05 * *$ & 121.08 & 0.46 & 18.84 & 39.09 & 84.27 \\
\hline Testers & 5 & $22.29 * *$ & $34.71 * *$ & $246.84 * *$ & $5.79 * *$ & $740.04 * *$ & 0.06 & $128.30 * *$ & 0.20 & $12.27 * *$ & 1.07 & $16.13 * *$ \\
\hline $\begin{array}{l}\text { Line } x \\
\text { Testers }\end{array}$ & 45 & $3.84 * *$ & $18.81 * *$ & $213.48 * *$ & $7.28 * *$ & $1565.43^{* *}$ & $0.06^{* * *}$ & $136.30 * *$ & $0.23 * *$ & $16.60 * *$ & $22.33 * *$ & $43.53 * *$ \\
\hline Error & 150 & 0.77 & 0.63 & 6.83 & 0.07 & 120.54 & 0.02 & 14.03 & 0.02 & 1.39 & $0.15 * *$ & 12.58 \\
\hline Total & 227 & 4.14 & 7.23 & 91.49 & 2.23 & 597.37 & 0.03 & 50.95 & 0.11 & 6.51 & 9.70 & 27.14 \\
\hline
\end{tabular}


Table.2 Performance of five superior crosses for each of eleven characters in sesame

\begin{tabular}{|c|c|c|c|c|c|c|c|c|c|}
\hline \multirow[t]{2}{*}{ Cross } & \multirow{2}{*}{$\begin{array}{c}\text { per se } \\
\text { performance }\end{array}$} & \multirow{2}{*}{$\begin{array}{c}\text { sca } \\
\text { effect }\end{array}$} & \multicolumn{2}{|c|}{ Parent 1} & \multicolumn{2}{|c|}{ Parent 2} & \multicolumn{3}{|c|}{ Heterosis over } \\
\hline & & & per se & gca effect & per se & gca effect & MP & BP & Swetha til \\
\hline \multicolumn{10}{|l|}{ Days to $50 \%$ flowering } \\
\hline SI-7818 x SI-3171 & 36.00 & $-1.85 * *$ & 37.00 & -0.217 & 38.33 & $-1.206^{* *}$ & $-4.42 * *$ & $-6.09 *$ & $-5.26 * *$ \\
\hline JCS-724 x IS 562 B & 36.00 & $-1.61 * *$ & 34.00 & $-1.494 * *$ & 40.00 & -0.172 & -2.70 & $-10.00 * *$ & $-5.26 *$ \\
\hline KMR-24 x IS 562 B & 36.00 & $-1.77 * *$ & 34.67 & -1 & 40.00 & -0.172 & $-3.57 *$ & $-10.00 * *$ & $-5.26^{*}$ \\
\hline JCS-724 x KMR-78 & 36.67 & -0.47 & 34.00 & $-1.328 * *$ & 38.00 & $-0.639 * *$ & 1.85 & -3.51 & -3.51 \\
\hline KMR-108 x SI-3171 & 37.00 & 0.04 & 36.00 & $-1.494 * *$ & 38.33 & $-1.206 * *$ & -0.45 & -3.48 & -2.63 \\
\hline \multicolumn{10}{|l|}{ Days to maturity } \\
\hline SI-7818 x TKG-22 & 90.33 & $-3.54 * *$ & 94.67 & $-1.106 * *$ & 94.33 & -0.239 & $-4.41 * *$ & $-4.58 * *$ & $-3.90 * *$ \\
\hline KMR-24 x KMR-78 & 90.67 & $-3.38 * *$ & 93.67 & $-0.694 * *$ & 94.33 & $-1.006 * *$ & $-3.55 * *$ & $-3.89 * *$ & $-3.55 * *$ \\
\hline JCS-720 x SI-3171 & 90.67 & $-2.51 * *$ & 91.00 & 0.25 & 91.67 & $-1.272 * *$ & -0.073 & -1.09 & $-3.55 * *$ \\
\hline KMR-24 x IS 562 B & 91.00 & $-4.12 * *$ & 93.60 & -0.361 & 99.00 & 0.061 & $-5.54 * *$ & $-8.08 * *$ & $-3.19 *$ \\
\hline CST 2001-5 x SI-3171 & 91.33 & -0.56 & 94.00 & 0.25 & 91.67 & $-1.272 * *$ & -1.62 & $-2.84 *$ & $-2.84 *$ \\
\hline \multicolumn{10}{|l|}{ Plant height } \\
\hline S-0018 x TKG-22 & 98.00 & $-14.77 * *$ & 131.67 & $-1.639 * *$ & 117.6 & $-2.43 * *$ & $-22.30 * *$ & $-27.23 * *$ & $-24.23 * *$ \\
\hline KMR-108 x SI-3171 & 99.33 & $-15.37 * *$ & 128.13 & $-9.493 * *$ & 116.63 & $-1.813 * *$ & $-18.83 * *$ & $-22.48 * *$ & $-23.20 * *$ \\
\hline PKDS-62 x KMR-78 & 99.67 & $-22.49 * *$ & 125.47 & $-8.181 * *$ & 130.07 & $-2.566 * *$ & $-21.99 * *$ & $-23.37 * *$ & $-22.94 * *$ \\
\hline IS 1547 A x SI-3171 & 109.13 & $-7.21 * *$ & 129.43 & 0.029 & 116.63 & $-1.813 * *$ & $-11.30 * *$ & $-15.68 * *$ & $-15.62 * *$ \\
\hline JCS-724 x KMR-78 & 109.87 & $-15.91 * *$ & 129.27 & $-6.537 * *$ & 130.07 & $-2.566 * *$ & $-15.27 * *$ & $-15.53 * *$ & $-15.05 * *$ \\
\hline \multicolumn{10}{|l|}{$\begin{array}{l}\text { No. of effective } \\
\text { primaries/plant }\end{array}$} \\
\hline S-0018 x KMR-78 & 8.33 & $1.23 * *$ & 5.83 & $0.373 * *$ & 6.20 & $-0.383 * *$ & $38.50 * *$ & $34.41 * *$ & $5.93 *$ \\
\hline PKDS-62 x IS 562 B & 8.00 & $4.55^{* *}$ & 5.53 & $0.707 * *$ & 6.33 & $0.297 * *$ & $103.21 * *$ & $82.69 * *$ & $61.02 * *$ \\
\hline SI-7818 x SI-3171 & 7.80 & $3.82 * *$ & 7.20 & $0.557 * *$ & 6.00 & 0.05 & $99.42 * *$ & $81.15 * *$ & $46.61 * *$ \\
\hline KKS-98049 x SI-3171 & 7.70 & $1.41 * *$ & 6.97 & $1.034 * *$ & 6.00 & 0.05 & $44.00 * *$ & $37.80 * *$ & $22.03 * *$ \\
\hline KKS-98049 x IS 562 B & 7.67 & 0.23 & 6.97 & $1.034 * *$ & 6.33 & $0.297 * *$ & $24.70 * *$ & $24.40 * *$ & $10.17 * *$ \\
\hline
\end{tabular}


Table.2 continued...

\begin{tabular}{|c|c|c|c|c|c|c|c|c|c|}
\hline \multirow[t]{2}{*}{ Cross } & \multirow{2}{*}{$\begin{array}{c}\text { per se } \\
\text { performance }\end{array}$} & \multirow[t]{2}{*}{ sca effect } & \multicolumn{2}{|c|}{ Parent 1} & \multicolumn{2}{|c|}{ Parent 2} & \multicolumn{3}{|c|}{ Heterosis over } \\
\hline & & & per se & gca effect & per se & gca effect & MP & BP & Swetha til \\
\hline \multicolumn{10}{|l|}{$\begin{array}{l}\text { No. of effective } \\
\text { capsules/plant }\end{array}$} \\
\hline KMR-108 x JCS-507 & 152.50 & $48.94 * *$ & 118.73 & $7.716 * *$ & 126.73 & $5.989 *$ & $37.24 * *$ & $31.11 * *$ & $30.20 * *$ \\
\hline PKDS-62 x IS 562 B & 149.90 & $38.41 * *$ & 120.07 & $14.261 * *$ & 110.93 & -2.621 & $35.85 * *$ & $33.90 * *$ & $22.22 * *$ \\
\hline SI-7818 x KMS 5-396 & 146.27 & $27.21 * *$ & 85.62 & $13.144 * *$ & 86.00 & $4.066^{*}$ & $99.63 * *$ & $95.07 * *$ & $18.65 * *$ \\
\hline SI-7818 x SI-3171 & 144.27 & $24.53 * *$ & 85.62 & $13.144 * *$ & 98.33 & 0.739 & $62.61 * *$ & $38.02 * *$ & $14.85 *$ \\
\hline KKS-98049 x SI-3171 & 143.90 & $26.17 * *$ & 125.03 & $10.144 * *$ & 98.33 & 0.739 & $27.88 * *$ & $19.91 * *$ & $13.98^{*}$ \\
\hline \multicolumn{10}{|l|}{ Capsule length } \\
\hline PKDS-62 x IS 562 B & 3.00 & $0.42 * *$ & 2.36 & $0.097 * *$ & 2.42 & 0.015 & $25.49 * *$ & $23.93 * *$ & $12.48 * *$ \\
\hline SI-7818 x SI-3171 & 2.84 & $0.29 * *$ & 2.53 & 0.032 & 2.55 & 0.044 & $11.88 * *$ & $11.37 * *$ & 6.37 \\
\hline S-0018 x SI-3171 & 2.74 & $0.21 * *$ & 2.36 & 0.015 & 2.55 & 0.044 & $11.82 * *$ & 7.58 & 2.75 \\
\hline IS 1547 A x TKG-22 & 2.69 & $0.19 * *$ & 2.44 & -0.023 & 2.39 & 0.045 & $11.25^{* *}$ & $9.96 *$ & 0.62 \\
\hline JCS-720 x KMS 5-396 & 2.62 & $0.20 * *$ & 2.53 & -0.048 & 2.22 & 0.005 & $10.46^{* *}$ & 3.55 & -1.75 \\
\hline \multicolumn{10}{|l|}{ No .of seeds per capsule } \\
\hline KMR-24 x KMS 5-396 & 76.47 & $10.12 * *$ & 65.93 & $2.109 *$ & 69.97 & $2.238 * *$ & $12.53 * *$ & $9.29 *$ & $26.59 * *$ \\
\hline JCS-724 x SI-3171 & 75.93 & $12.66 * *$ & 63.30 & $4.365 * *$ & 55.27 & $-3.089 * *$ & $28.09 * *$ & $19.96 * *$ & $25.71 * *$ \\
\hline KMR-24 x KMR-78 & 73.33 & $6.80 * *$ & 65.93 & $2.109 *$ & 69.07 & $2.418 * *$ & $8.64 *$ & 6.18 & $21.41 * *$ \\
\hline KKS-98049 x KMR-78 & 72.87 & $10.18 * *$ & 53.53 & -1.735 & 69.07 & $2.418 * *$ & $18.87 * *$ & 5.50 & $20.63 * *$ \\
\hline CST 2001-5 x IS 562 B & 71.33 & $6.13 * *$ & 63.60 & $3.398 * *$ & 59.07 & -0.195 & $16.30 * *$ & $12.16^{*}$ & $18.10 * *$ \\
\hline \multicolumn{10}{|l|}{1000 seed weight } \\
\hline JCS-720 x KMS 5-396 & 3.77 & $1.00 * *$ & 2.66 & 0.068 & 2.54 & $0.149 * *$ & $44.74 * *$ & $41.46^{* *}$ & $55.02 * *$ \\
\hline CST 2001-5 x IS 562 B & 3.53 & $0.54 * *$ & 3.17 & $0.394 * *$ & 2.45 & 0.037 & $25.61 * *$ & $11.41 * *$ & $45.20 * *$ \\
\hline CST 2001-5 x TKG-22 & 3.13 & $0.21 * *$ & 3.17 & $0.394 * *$ & 3.27 & -0.035 & -2.84 & -4.38 & $28.72 * *$ \\
\hline $\begin{array}{l}\text { CST 2001-5 x KMS 5- } \\
396\end{array}$ & 3.11 & 0.02 & 3.17 & $0.394 * *$ & 2.54 & $0.149 * *$ & $9.05^{*}$ & -1.70 & $18.11 * *$ \\
\hline KKS-98049 x JCS-507 & 3.07 & $0.51 * *$ & 2.62 & 0.055 & 2.51 & -0.045 & $19.80 * *$ & $17.12 * *$ & $26.47 * *$ \\
\hline
\end{tabular}


Table.2 continued...

\begin{tabular}{|c|c|c|c|c|c|c|c|c|c|}
\hline \multirow[t]{2}{*}{ Cross } & \multirow{2}{*}{$\begin{array}{c}\text { per se } \\
\text { performance }\end{array}$} & \multirow[t]{2}{*}{ sca effect } & \multicolumn{2}{|c|}{ Parent 1} & \multicolumn{2}{|c|}{ Parent 2} & \multicolumn{3}{|c|}{ Heterosis over } \\
\hline & & & perse & gca effect & per se & gca effect & MP & BP & Swetha til \\
\hline \multicolumn{10}{|l|}{ Seed yield/plant } \\
\hline PKDS-62 x IS 562 B & 18.24 & $6.33 * *$ & 12.37 & $-0.683^{*}$ & 10.43 & -0.192 & $59.99 * *$ & $47.41 * *$ & $19.51 * *$ \\
\hline KKS-98049 x KMR-78 & 18.17 & $4.54 * *$ & 13.38 & $0.749 * *$ & 12.81 & 0.100 & $38.71 * *$ & $35.73 * *$ & $19.04 * *$ \\
\hline SI-7818 x SI-3171 & 17.99 & $3.23 * *$ & 11.38 & $1.128 * *$ & 8.70 & $0.850 * *$ & $79.24 * *$ & $58.11 * *$ & $17.90 * *$ \\
\hline CST 2001-5 x TKG-22 & 17.69 & $3.85 * *$ & 12.11 & $1.949 * *$ & 12.62 & $-0.887 * *$ & $43.08 * *$ & $40.22 * *$ & $15.94 * *$ \\
\hline KKS-98049 x SI-3171 & 17.45 & $3.07 * *$ & 13.38 & $0.749 * *$ & 8.70 & $0.850 * *$ & $58.07 * *$ & $30.38 * *$ & $14.35 *$ \\
\hline \multicolumn{10}{|l|}{ Oil content } \\
\hline SI-7818 x SI-3171 & 51.60 & $9.18 * *$ & 47.5 & $1.403 * *$ & 48.3 & $-0.261 * *$ & $7.76^{* *}$ & $6.91 * *$ & $-1.28 *$ \\
\hline KMR-108 x KMR-78 & 45.40 & $4.61 * *$ & 45.0 & $-0.714 * *$ & 40.4 & $0.186^{*}$ & $6.33 * *$ & 0.89 & $-13.20 * *$ \\
\hline CST 2001-5 x TKG-22 & 44.50 & $1.41 * *$ & 44.4 & $1.569 * *$ & 35.5 & $0.209 *$ & $11.35^{* *}$ & 0.15 & $-14.92 * *$ \\
\hline CST 2001-5 x KMR-78 & 44.50 & $1.50 * *$ & 44.4 & $1.569 * *$ & 40.4 & $0.186^{*}$ & $5.07 * *$ & 0.30 & $-14.80 * *$ \\
\hline PKDS-62 x JCS-507 & 44.40 & $3.30 * *$ & 44.5 & -0.053 & 42.3 & -0.094 & $2.30 * *$ & -0.22 & $-14.99 * *$ \\
\hline \multicolumn{10}{|l|}{ Chlorophyll content } \\
\hline IS 1547 A x SI-3171 & 55.80 & $4.22 * *$ & 45.99 & $2.986 * *$ & 41.55 & $1.360^{*}$ & $27.47 * *$ & $21.32 * *$ & $29.77 * *$ \\
\hline S-0018 x KMR-78 & 54.23 & $4.70^{*}$ & 47.23 & $3.167 * *$ & 45.64 & -0.862 & $16.79 * *$ & $14.82 *$ & $26.12 * *$ \\
\hline KMR-108 x TKG-22 & 53.36 & $4.85^{*}$ & 41.51 & 1.287 & 41.94 & -0.003 & $27.86^{* *}$ & $27.19 * *$ & $24.09 * *$ \\
\hline S-0018 x JCS-507 & 53.30 & 3.12 & 47.23 & $3.167 * *$ & 38.42 & -0.213 & $24.46^{* *}$ & $12.84 *$ & $23.95 * *$ \\
\hline JCS-724 x SI-3171 & 52.80 & $5.31 *$ & 41.42 & -1.096 & 41.55 & $1.360 *$ & $27.27 * *$ & $27.07 * *$ & $22.79 * *$ \\
\hline
\end{tabular}


Table.3 Heterosis over mid parent (MP), better parent (BP), check and sca effects of superior ten crosses for seed yield and other component characters in sesame

\begin{tabular}{|c|c|c|c|c|c|c|c|c|c|c|c|c|c|}
\hline Cross & perse & $\begin{array}{c}\text { Heterosis } \\
\text { \&sca }\end{array}$ & $\begin{array}{c}\text { Days to } \\
50 \% \\
\text { flowering }\end{array}$ & $\begin{array}{l}\text { Days to } \\
\text { maturity }\end{array}$ & $\begin{array}{c}\text { Plant } \\
\text { height } \\
(\mathrm{cm})\end{array}$ & $\begin{array}{c}\text { No. of } \\
\text { effective } \\
\text { primaries } \\
\text { / plant }\end{array}$ & $\begin{array}{l}\text { No. of } \\
\text { effective } \\
\text { capsules/ } \\
\text { plant }\end{array}$ & $\begin{array}{l}\text { Capsule } \\
\text { length } \\
\text { (cm) }\end{array}$ & $\begin{array}{c}\text { No. of } \\
\text { seeds/ } \\
\text { capsule }\end{array}$ & $\begin{array}{l}\text { 1000-seed } \\
\text { weight } \\
\text { (g) }\end{array}$ & $\begin{array}{c}\text { Seed } \\
\text { yield/ } \\
\text { plant }(g)\end{array}$ & $\begin{array}{c}\text { Oil } \\
\text { content } \\
(\%)\end{array}$ & $\begin{array}{c}\text { Chlorophyll } \\
\text { content } \\
\text { (SPAD Units) }\end{array}$ \\
\hline \multirow[t]{4}{*}{ PKDS-62 x IS 562 B } & 18.24 & MP & -3.39 & $-5.02 * *$ & 5.12 & $103.21 * *$ & $35.58 * *$ & $25.49 * *$ & 6.72 & $16.87 * *$ & $59.99 * *$ & $6.75 * *$ & 10.33 \\
\hline & & $\mathrm{BP}$ & $-5.00 *$ & $-5.65 * *$ & 2.39 & $82.69 * *$ & $33.90 * *$ & $23.93 * *$ & 5.30 & 8.32 & $47.41 * *$ & $-6.51 * *$ & -7.95 \\
\hline & & $\mathrm{SH}$ & 0.00 & 0.71 & 4.77 & $61.02 * *$ & $22.22 * *$ & $12.48 * *$ & 2.97 & 9.24 & $19.51 * *$ & $-20.34 * *$ & $19.60 * *$ \\
\hline & & sca & $-1.11 *$ & -0.45 & $8.05 * *$ & $4.55^{* *}$ & $38.41 * *$ & $0.42 * *$ & -0.62 & $0.19 *$ & $6.33 * *$ & $0.53 *$ & $4.36 *$ \\
\hline \multirow[t]{4}{*}{ KKS-98049 x KMR-78 } & 18.17 & MP & 2.93 & 0.36 & -3.04 & $27.59 * *$ & -5.06 & -3.58 & $18.87 * *$ & -5.51 & $38.71 * *$ & $-3.71 * *$ & 2.21 \\
\hline & & $\mathrm{BP}$ & -1.60 & -1.06 & -5.01 & $20.57 * *$ & -6.69 & -7.03 & 5.50 & $-15.22 * *$ & $35.73 * *$ & $-7.79 * *$ & 1.97 \\
\hline & & SH & $7.89 * *$ & -0.71 & -0.41 & $6.78^{*}$ & $-11.30^{*}$ & $-10.86^{* *}$ & $20.63 * *$ & $15.24 * *$ & $19.04 * *$ & $-22.19 * *$ & 8.74 \\
\hline & & sca & $1.36^{* *}$ & -0.72 & 1.62 & $0.64 * *$ & $-12.79 *$ & 0.00 & $10.18 * *$ & $0.24 * *$ & $4.54 * *$ & $-0.48 *$ & 1.92 \\
\hline \multirow[t]{4}{*}{ SI-7818 x SI-3171 } & 17.99 & MP & -4.42 & 1.61 & $13.37 * *$ & $99.42 * *$ & $62.61 * *$ & $11.88 * *$ & $10.45^{*}$ & 6.57 & $79.24 * *$ & $7.76 * *$ & 4.74 \\
\hline & & $\mathrm{BP}$ & $-6.09 *$ & 0.00 & $10.26^{* *}$ & $81.15^{* *}$ & $38.02 * *$ & $11.37 * *$ & 3.36 & 4.75 & $58.11 * *$ & $6.91 * *$ & 3.69 \\
\hline & & SH & $-5.26^{*}$ & 0.71 & -0.57 & $46.61 * *$ & $14.58^{*}$ & 6.37 & 8.49 & 6.50 & $17.90 * *$ & $-1.28 *$ & 0.20 \\
\hline & & $s c a$ & $-1.85^{* *}$ & $1.83 * *$ & -1.56 & $3.82 * *$ & $24.53 * *$ & $0.29 * *$ & $7.73 * *$ & 0.06 & $3.23 * *$ & $9.18 * *$ & -2.29 \\
\hline \multirow[t]{4}{*}{ CST 2001-5 x TKG-22 } & 17.69 & MP & 0.00 & $-3.01 * *$ & 5.82 & $9.71 * *$ & $23.07 * *$ & -3.33 & -1.86 & -2.84 & $43.08 * *$ & $11.35^{* *}$ & 4.79 \\
\hline & & $\mathrm{BP}$ & 0.00 & $-3.18^{*}$ & 3.12 & -3.42 & $16.83 *$ & $-9.20 *$ & -5.87 & -4.38 & $40.22 * *$ & 0.15 & 0.80 \\
\hline & & $\mathrm{SH}$ & 3.51 & $-2.84^{*}$ & -1.19 & -4.24 & -0.91 & -7.62 & 7.94 & $28.72 * *$ & $15.94 *$ & $-14.92 * *$ & 6.46 \\
\hline & & sca & -0.99 & $-1.59 * *$ & 0.31 & $1.42 * *$ & $26.02 * *$ & -0.10 & 0.42 & $0.21 *$ & $3.85 * *$ & $1.41 * *$ & -0.25 \\
\hline \multirow[t]{4}{*}{ KKS-98049 x SI-3171 } & 17.45 & MP & $-5.00 *$ & 0.00 & 0.86 & $44.00 * *$ & $27.88 * *$ & -2.15 & 8.58 & -0.84 & $58.07 * *$ & $-10.36 * *$ & $19.41 * *$ \\
\hline & & $\mathrm{BP}$ & $-8.80 * *$ & 0.00 & -6.19 & $37.80 * *$ & $19.91 * *$ & -2.34 & 6.88 & -5.33 & $30.38 * *$ & $-14.23 * *$ & $13.80 *$ \\
\hline & & SH & 0.00 & -2.48 & 1.65 & $22.03 * *$ & $13.98^{*}$ & -6.34 & -2.21 & 2.22 & $14.35 *$ & $-20.79 * *$ & $21.36 * *$ \\
\hline & & sca & $-1.07 *$ & $-2.12 * *$ & -0.73 & $1.41 * *$ & $26.17 * *$ & 0.03 & 1.89 & -0.06 & $3.07 * *$ & 0.7099 & $5.12 *$ \\
\hline
\end{tabular}


Table.3 continued...

\begin{tabular}{|c|c|c|c|c|c|c|c|c|c|c|c|c|c|}
\hline Cross & perse & $\begin{array}{l}\text { Heterosis } \\
\text { \&sca }\end{array}$ & $\begin{array}{l}\text { Days to } \\
50 \% \\
\text { flowering }\end{array}$ & $\begin{array}{l}\text { Days to } \\
\text { maturity }\end{array}$ & $\begin{array}{l}\text { Plant } \\
\text { height } \\
(\mathbf{c m})\end{array}$ & $\begin{array}{c}\text { No. of } \\
\text { effective } \\
\text { primaries } \\
\text { / plant }\end{array}$ & $\begin{array}{l}\text { No. of } \\
\text { effective } \\
\text { capsules/ } \\
\text { plant }\end{array}$ & $\begin{array}{l}\text { Capsule } \\
\text { length } \\
\text { (cm) }\end{array}$ & $\begin{array}{l}\text { No. of } \\
\text { seeds/ } \\
\text { capsule }\end{array}$ & $\begin{array}{l}\text { 1000-seed } \\
\text { weight } \\
\text { (g) }\end{array}$ & $\begin{array}{c}\text { Seed } \\
\text { yield/plant } \\
\text { (g) }\end{array}$ & $\begin{array}{c}\text { Oil } \\
\text { content } \\
(\%)\end{array}$ & $\begin{array}{l}\text { Chlorophyll } \\
\text { content } \\
\text { (SPAD Units) }\end{array}$ \\
\hline \multirow[t]{4}{*}{ KMR-24 x KMR-78 } & 16.90 & MP & $7.34 * *$ & $-3.55 * *$ & 5.34 & $26.38 * *$ & 7.06 & 5.07 & $8.64 *$ & -6.15 & $53.90 * *$ & $2.55 * *$ & -1.78 \\
\hline & & BP & 2.63 & $-3.89 * *$ & 5.18 & $17.20 * *$ & 5.87 & 3.23 & 6.18 & $-16.40 * *$ & $31.91 * *$ & 0.48 & -7.34 \\
\hline & & SH & 2.63 & $-3.55 * *$ & 5.77 & $-7.63 * *$ & -2.81 & $-8.11 *$ & $21.41 * *$ & $13.63^{*}$ & 10.73 & $-19.13 * *$ & 10.91 \\
\hline & & $s c a$ & $1.69 * *$ & $-3.38 * *$ & $9.41 * *$ & $1.13 * *$ & $14.56^{*}$ & 0.03 & $6.80 * *$ & $0.36^{* *}$ & $4.71 * *$ & -0.35 & 3.23 \\
\hline \multirow{4}{*}{$\begin{array}{c}\text { CST 2001-5 x KMS 5- } \\
396\end{array}$} & 16.72 & MP & $4.68 *$ & -0.36 & 3.95 & $-25.00 * *$ & $42.58^{* *}$ & 5.41 & -3.07 & $9.05^{*}$ & $67.58 * *$ & $-10.15^{* *}$ & 5.28 \\
\hline & & $\mathrm{BP}$ & 4.24 & -0.71 & -0.47 & $-27.95 * *$ & $28.18 * *$ & -4.29 & -7.48 & -1.70 & $37.99 * *$ & $-11.96 * *$ & -0.65 \\
\hline & & SH & $7.89 * *$ & -0.71 & 4.23 & $-22.46^{* *}$ & -2.30 & -2.62 & 7.17 & $28.11 * *$ & 9.54 & $-22.07 * *$ & 4.93 \\
\hline & & $s c a$ & $1.14^{*}$ & -0.86 & $5.03 * *$ & $-0.79 * *$ & 11.79 & 0.07 & -2.90 & 0.02 & $1.43^{*}$ & $-2.20 * *$ & -0.70 \\
\hline \multirow{4}{*}{$\begin{array}{c}\text { IS-1547 A x KMS 5- } \\
396\end{array}$} & 16.48 & MP & -1.60 & $3.16^{* *}$ & -5.16 & $15.91 * *$ & $51.73 * *$ & $11.59 * *$ & -6.65 & $-9.07 *$ & $68.99 * *$ & $-10.79 * *$ & 10.55 \\
\hline & & $\mathrm{BP}$ & $-7.52 * *$ & 1.38 & $-7.26^{*}$ & -3.94 & $35.73 * *$ & 6.41 & $-11.39 *$ & $-13.76^{* *}$ & $41.26 * *$ & $-12.46 * *$ & 3.71 \\
\hline & & SH & $7.89 * *$ & $4.26 * *$ & -2.89 & 3.39 & 4.64 & -2.62 & 2.64 & 0.60 & 7.97 & $-22.51 * *$ & 10.93 \\
\hline & & $s c a$ & 0.25 & 0.53 & $7.59 * *$ & $0.80 * *$ & $35.92 * *$ & $0.15^{*}$ & 0.04 & -0.10 & $4.00 * *$ & $2.44 * *$ & -2.31 \\
\hline \multirow[t]{4}{*}{ KMR-108 x SI-3171 } & 15.96 & MP & -0.45 & 0.53 & $-18.83 * *$ & 1.75 & $16.91 * *$ & 5.16 & 2.38 & $15.02 * *$ & $33.22 * *$ & $-10.19 * *$ & $22.75 * *$ \\
\hline & & $\mathrm{BP}$ & -3.48 & -1.74 & $-22.48 * *$ & -2.86 & 12.24 & -1.44 & 1.33 & $14.11^{* *}$ & 4.55 & $-11.46^{* *}$ & $20.96 * *$ \\
\hline & & SH & -2.63 & 0.35 & $-23.20 * *$ & $-13.56^{* *}$ & 1.50 & -5.87 & -7.29 & $12 . .04 *$ & 4.60 & $-21.62 * *$ & $16.78^{*}$ \\
\hline & & $s c a$ & 0.04 & $-0.95 *$ & $-15.37 * *$ & $-0.46^{* *}$ & 8.89 & 0.07 & 0.08 & $0.34 * *$ & $2.29 * *$ & $-6.81 * *$ & 0.31 \\
\hline \multirow[t]{4}{*}{ S-0018 x KMR-78 } & 15.71 & MP & $-7.50 * *$ & $-3.66^{* *}$ & $-9.29 * *$ & $38.50 * *$ & -3.45 & 0.56 & $-11.68 * *$ & $-17.68 * *$ & $22.27 * *$ & 0.97 & $16.79 * *$ \\
\hline & & BP & $-11.90 * *$ & $-4.83^{* *}$ & $-10.84 * *$ & $34.44 * *$ & -4.99 & 0.14 & $-12.07 * *$ & $-25.83 * *$ & $21.95^{* *}$ & -1.49 & $14.82 *$ \\
\hline & & $\mathrm{SH}$ & -2.63 & -2.13 & $-7.16^{*}$ & $5.93^{*}$ & -9.90 & $-10.86^{* *}$ & 0.55 & 0.82 & 2.92 & $-23.92 * *$ & $26.12 * *$ \\
\hline & & sca & $-2.42 * *$ & -0.88 & $7.43 * *$ & $1.23 * *$ & 4.15 & -0.06 & -1.24 & -0.02 & $2.52 * *$ & $-1.45 * *$ & $4.70 *$ \\
\hline
\end{tabular}


Table.4 Number of crosses with significant desirable heterosis for eleven characters in Sesame

\begin{tabular}{lcccc}
\hline Character & sca effect & \multicolumn{3}{c}{ Significant heterosis in the desired direction over } \\
\cline { 3 - 6 } & Mid parent & Better parent & Standard check & ( Swetha til) \\
\hline 1.Days to 50 \% flowering & 11 & 11 & 25 & 3 \\
2. Days to maturity & 20 & 16 & 27 & 7 \\
3. Plant height & 19 & 24 & 33 & 8 \\
4. No.of effective primaries/plant & 23 & 31 & 24 & 12 \\
5. No.of effective capsules/plant & 10 & 25 & 12 & 5 \\
6. Capsule length & 7 & 12 & 5 & 1 \\
7. No.of seeds /capsule & 15 & 11 & 5 & 13 \\
8. 1000 seed weight & 12 & 7 & 5 & 12 \\
9. Seed yield/plant & 11 & 27 & 16 & 5 \\
10. Oil content & 24 & 21 & 7 & 0 \\
11. Chlorophyll content (SPAD) & 8 & 24 & 16 & 23 \\
& & & & \\
\hline
\end{tabular}

Significant positive heterosis over mid parent has been reported by Uzun et al., (2004), Mothilal and Ganesan (2005). While positive heterosis over better parent observed in agreement with earlier findings of Mothilal and Manoharan (2004), Mothilal and Ganesan (2005). The presence of standard heterosis for seed yield in few crosses is confirmed with the many workers viz., Mothilal and Manoharan (2004), Raghunaiah (2005).

For commercial point of view, the superiority of new hybrids for yield can be judged by comparing their performance with the best cultivated hybrid/s or variety. The Swetha til therefore used as standard check in order to obtain information regarding superiority of new hybrids over the best cultivated varieties. The highest yielding hybrid PKDS-62 x IS $562 \mathrm{~B}(18.24 \mathrm{~g})$ had the standard heterosis of 19.51 per cent and exhibited considerable amount of relative heterosis $(59.99 \%)$ and heterobeltiosis $(47.41 \%)$ and secured first position in respect of significant desirable sca effect (6.33) (Table 2)

In the present study, where the per se performance of the parents and the per cent heterosis of the resultant hybrids were considered with best ten crosses mentioned (Table 2), the hybrids PKDS-62 x IS $562 \mathrm{~B}$ and KKS-98049 x KMR-78 exhibited high per se performance for yield with considerable levels of heterosis, where parents involved in these crosses recorded substantial yield levels, whereas the hybrid resulting from low yielding parents, SI-7818 and KMS 5-396 exhibited substantial increase in yield with highest percent of relative heterosis and heterobeltiosis. Such situation could be attributable to high inter-allelic interaction canceling the individual effects of each other.

The cross SI-7818 x SI-3171 recorded significant heterosis for oil content in positive direction which also recorded considerable positive heterotic values for yield. For number 
of effective capsules per plant and seed yield, SI-7818 x KMS 5-396, SI-7818 x SI-3171, IS 1547 A x KMS 5-396, CST 2001-5 x KMS 5396 and PKDS-62 x IS 562 B exhibited significant positive heterosis. Whereas the crosses KKS-98049 x KMR-78, JCS-724 x SI-3171, KMR-24 x KMS 5-396 and CST 2001-5 x JCS-507 exhibited significant positive heterosis for number of seeds per capsules and seed yield per plant. The crosses SI-7818 x KMS 5-396, KKS-98049 x JCS507, JCS-720 x KMS 5-396, KMR-108 x SI3171recorded significant heterosis for 1000 seed weight in positive direction which also recorded considerable positive heterosis values for yield. So these crosses can be exploited to improve the yield along with one or more traits through heterosis breeding.

In conclusion, the hybrids PKDS-62 $\mathrm{x}$ IS 562 $B$ and KKS-98049 $x$ KMR-78 which exhibited significant heterosis for yield were found to be early as they recorded significant heterosis in desired direction. SI-7818 x SI3171, PKDS-62 x IS 562 B and KKS-98049 x SI-3171 which recorded high heterotic values for seed yield also exhibited high values for its important yield component traits such as effective capsules per plant, number of effective primaries per plant and capsule length.

\section{References}

Damodaram T, Hegde DM (2010) Oil seeds situation: A statistical compendium. Directorate of Oilseeds Research, Hyderabad.

Deepa SP, Ananda KCR (2001) Heterosis for yield and yield components in sesame (Sesamum indicum L.). Sesame and Safflower Newsletter, 16: 6-8.

Karad SR, Dusane SM, Surve US (2002) Studies on exploitation of useful heterosis in sesamum. Journal of Maharashtra Agricultural University 27:
57-59.

Kempthorne O. (1957) An introduction to genetic statistics. John Wiley and Sons, New York. pp. 545.

Mishra AK, Sikarwar RS (2001) Heterosis and combining ability analysis in sesame. Sesame and Safflower Newsletter, 16: 1-5.

Mosjidis JA (1982). The inheritance of oil content and its fatty acid composition in the sesame seed. (Sesamum indicum L.) and the study of their correlations. Diss Abstr Intermat B., 42: 3947B.

Mothilal A Manoharan V (2004) Heterosis and combining ability in sesame (Sesamum indicum L.). Crop Research 27: 282-287.

Mothilal A, Ganesan KN (2005) Heterosis studies in sesame (Sesamum indicum L.). Agricultural Science Digest 25 (1): 74-76.

Neelima S, Chengareddy V, Narisireddy A (2004) Combining ability analysis and inheritance of fibre quality traits in American cotton (Gossypium hirsutum L.). Madras Agricultural Journal 91 (712): 518-521.

Nijagun HG, Parameswarappa KG, Muralikrishna S, Salimath PM (2003). Implication of non-additive genetic effects and heterosis and breeding hybrids in sesamum (Sesamum indicum L.). National Journal of Plant Improvement 5: 26-30.

Panse VG, Sukhatme PV, (1985) Statistical methods for agricultural workers, Indian Council of Agricultural Research, New Delhi.

Raghunaiah E (2005) Studies on heterosis, combining ability and character association in sesame (Sesamum indicum L.). M.Sc. (Ag.) Thesis, Acharya N.G. Ranga Agricultural University, Hyderabad.

Rauf S, Munir H, Basra SMA, Abdullojon E (2006) Combining ability analysis in 
upland cotton (Gossypium hirsutum L.). International Journal of Agriculture and Biology8 (3): 341-343.

RaySD, Sen S (1992) Heterosis in sesame (Sesamum indicum L.). Tropical Agricculture 69 (3): 276-278.

SaravananS, Nadarajan N (2002) Studies on heterosis in sesame (Sesamum indicum
L.). Indian Journal of Genetics62 (3): 271-272.

Uzun B, Ozbas MO, Canci H, Cagirgan MI (2004) Heterosis for agronomic traits in sesame hybrids of cultivars $\mathrm{x}$ closed capsule mutants. Acta Agriculturae Scandinavica. Section B, Soil and Plant Science 54: 2, 108-112.

\section{How to cite this article: \\ Jawahar Lal, J., Kuldeep Singh Dangi and Sudheer Kumar, S. 2020. Genetic Analysis of Superior Crosses for Quantitative Traits in Sesame (Sesamum indicum L.). Int.J.Curr.Microbiol.App.Sci. 9(01): 2564-2576. doi: https://doi.org/10.20546/ijcmas.2020.901.291}

